\title{
Postcopulatory, prezygotic isolation in flour beetles
}

\author{
MICHAEL J. WADE*, HAROLD PATTERSON $\dagger$, NANCY W. CHANG \& NORMAN A. \\ JOHNSON \\ Department of Ecology and Evolution, 1101 E. 57th Street, University of Chicago, Chicago, IL 60637 and tHyde Park \\ Career Academy, 6220 S. Stony Is/and, Chicago, IL 60637, USA
}

\begin{abstract}
We report the existence of postmating but prezygotic reproductive isolation within flour beetles of the genus Tribolium. Specifically, when a female of either $T$. castaneum or $T$. freemani is paired simultaneously with both a conspecific and a heterospecific male, virtually all of the offspring are sired by the conspecific male. In contrast, when a female of either species is paired only with a heterospecific male, she produces near normal numbers of offspring. Mate choice experiments rule out the possibility that premating reproductive isolation accounts for this phenomenon. A number of different mechanisms could explain this phenomenon of postmating but prezygotic reproductive isolation.
\end{abstract}

Keywords: speciation, postmating reproductive isolation, prezygotic reproductive isolation, mate choice, reinforcement, Tribolium.

\section{Introduction}

The process of speciation, the acquisition of genetic reproductive isolation between populations, is generally attributed to the gradual accumulation of genes as a by-product of other adaptive or neutral changes occurring in allopatry (Dobzhansky, 1937; Muller, 1942; Mayr, 1963; Charlesworth et al., 1987; Coyne, 1992; Wu \& Davis, 1993). Dobzhansky (1940) presented the 'reinforcement' model, in which natural selection plays a direct role in establishing premating reproductive isolation between populations which have established partial but not complete postmating reproductive isolation (presumably by the pleiotropic effects of other adaptive or neutral changes). The importance of the role of reinforcement in natural populations is controversial (Paterson, 1978; Littlejohn, 1981; Butlin, 1987, 1989; Coyne \& Orr, 1989; Howard, 1993).

Tribolium castaneum is a human-commensal flour beetle found worldwide in grain and other stored products. The general biology of this species is summarized by Sokoloff (1974). T. freemani is a closely related species to $T$. castaneum based on morphological, genetic, karyotypic and molecular data (Hinton, 1948; Sokoloff, 1974; Brownlee \& Sokoloff, 1988; Juan et al., 1993). These species are capable of producing large numbers of viable but sterile $F_{1}$ progeny on

*Correspondence. hybridization (Brownlee \& Sokoloff, 1988; Wade \& Johnson, 1993).

Within $T$. castaneum, mating occurs without elaborate courtship and the average copulation duration is 45 s (Park , 1933; Shrode, 1960; Sokoloff, 1974) but has not been described in T. freemani. Both sexes of $T$. castaneum will mate several times an hour (Park, 1933) and males will 'attempt copulation with other males, dead beetles of both sexes, or with any object, such as a lump of flour or frass, which looks like a beetle' (Sokoloff, 1974, p. 201). Thus, little premating reproductive isolation may be expected between these species.

\section{Materials and methods}

We used two different $T$. castaneum laboratory strains in these experiments: c-SM and c-pl. The c-SM (castaneum Standard Mixture) was established by M. Wade in 1973 by mass mating 12 males and 12 females from each of the four classic 'Park' T. castaneum stocks (see Park et al., 1964; Wade, 1976, 1977, for information about this strain). It has been maintained in large populations (of more than 1000 breeding adults) ever since. This stock also has alleles of an autosomal black body colour locus segregating (Wade \& Goodnight, 1991). The c-pl (castaneum pearl) strain has alleles segregating at both the black body colour locus and the pearl eye locus (see Park 1937, for a description of the 
pearl locus). Only one strain of $T$. freemani was available: in this strain there is no variation at any visible markers.

In the simultaneous male experiments, virgin males and females were isolated from $T$. freemani and the c-SM strain of $T$. castaneum. Two males and one virgin female were placed into a vial containing $8 \mathrm{~g}$ of fine sifted standard medium (by weight, 95 per cent stoneground whole wheat flour, 5 per cent dried brewers yeast, 0.03 per cent fumagillin; following the protocol of Wade \& Goodnight, 1991). There were six different treatments, representing all possible combinations of two males $(\mathrm{CC}, \mathrm{CF}$, or FF) and a single female (C or F) and six replicates of each treatment (six treatments $\times$ six replicates/treatment $=36$ vials total). Throughout this paper for the simultaneous male treatments, we will use the notation $\mathrm{CC}-\mathrm{F}$ to indicate that two $T$. castaneum males were mated to a single $T$. freemani female and similarly for the other five treatments. Each vial was fine sifted every 4 days for a total of 28 days. At each sift, all eggs were counted and transferred to a syracuse dish. The eggs were observed for hatching larvae daily for 8 or 9 days (i.e. until hatching had ceased). Hatching larvae were censused by replicate and transferred to a $8 \mathrm{~g}$ of regular medium to continue development. Vials with eggs and larvae were maintained in darkened incubators at $29^{\circ} \mathrm{C}$ and 70 per cent relative humidity throughout the period of egg and larvae collection. After 45 days, the adult progeny maturing from each replicate's larval collection was censused. Comparison of larval and adult numbers permits us to estimate survivorship.

The fertility of the adult progeny of the CC-F, FF-C, $\mathrm{CF}-\mathrm{C}$, and $\mathrm{CF}-\mathrm{F}$ treatments was tested by mating individual female progeny with three males from the species of the original mother (e.g. the CC-F female progeny were crossed to $T$. freemani males). The male progeny were individually mated to a single female from the original mother's species.

During the egg collections in the $\mathrm{CF}-\mathrm{C}$ and $\mathrm{CF}-\mathrm{F}$ treatments, it was not unusual to find females of both species engaged in interspecific copulations and copulations were commonly observed in both the CC-F and FF-C treatments. To quantify copulation rates, we observed the copulatory behaviour of 20 females of each species when presented simultaneously with one male of each species, i.e. 20 additional replicates of CF-C and CF-F were set up, each in a petri dish, each containing $1 \mathrm{~g}$ of flour. The elytra of the female were marked with silver paint to distinguish her from the males and the males of the different species were readily discriminated on the basis of body size differences, T. freemani being almost three times the mass of T. castaneum (Brownlee \& Sokoloff, 1988; Wade \&
Johnson, 1993). The total of 40 dishes was placed in a darkened incubator at $29^{\circ} \mathrm{C}$ and observed 28 times over 3 days; the identity of all copulating males was scored at each observation. The minimum time between observations was $20 \mathrm{~min}$ and there were a total of 1120 observations ( 20 females per species $\times 2$ species $\times 28$ observations per female $=1120$ observations).

\section{Results}

Table 1 illustrates the fecundity (numbers of eggs laid), the hatchability (proportion of eggs hatching) and the larval survival for the simultaneous male treatments. Note that the fecundity, hatchability and larval survivorship of a $T$. freemani female mated with two males of her own species (treatment FF-F) is equivalent to that of a $T$. freemani female mated with two $T$. castaneum males (treatment $\mathrm{CC}-\mathrm{F}$ ). However, the progeny resulting from the interspecific cross $(\mathrm{CC}-\mathrm{F})$ are nearly always sterile or, in rare instances (approximately 1 per cent) are weakly fertile, producing a few offspring (see also Wade \& Johnson, 1993).

When a $T$. freemani female is simultaneously presented with both a conspecific and a heterospecific male (treatment CF-F), the fecundity, hatchability and larval survivorship are again comparable to those of the conspecific (FF-F) or interspecific crosses $(\mathrm{CC}-\mathrm{F})$ (Table 1). Consequently, in the CF-F treatment, we would expect hybrids to constitute approximately half of the progeny ( 52.0 per cent, using the product of the relative fitnesses of the $\mathrm{FF}-\mathrm{F}$ and $\mathrm{CC}-\mathrm{F}$ treatments). The paternity tests of the CF-F progeny (the crosses to virgin $T$. freemani adults) permit conspecific and heterospecific progeny to be discriminated: intraspecific progeny are fertile while interspecific progeny are infertile. Of $191 \mathrm{CF}-\mathrm{F}$ offspring test crossed to virgin $T$. freemani adults, only six were sterile. As hybrids are nearly always completely sterile, this indicates that a maximum of 3.1 per cent of CF-F progeny are hybrids (6 of 191). This is more than a 16-fold difference between the expected and the observed proportions of hybrids $(P<0.00001, G$-test). (This estimate is conservative because ancillary data on body size and coloration indicate that two of the six sterile beetles were pure $T$. freemani.)

There is a similar discrepancy between the observed and the expected proportions of hybrids in the $\mathrm{CF}-\mathrm{C}$ treatment. Owing to the differences in fecundity between intraspecific matings $(\mathrm{CC}-\mathrm{C})$ and interspecific matings ( $\mathrm{FF}-\mathrm{C})$ and the lower hatchability of $\mathrm{FF}-\mathrm{C}$ eggs, the difference between observed and expected is smaller in this case but still highly significant $(P<0.010, G$-test $)$. 
Table 1 Fecundity, hatchability and larval survival for the simultaneous male treatments

\begin{tabular}{|c|c|c|c|c|c|c|}
\hline \multirow{2}{*}{$\frac{\text { Treatment }}{\text { males-female }}$} & \multicolumn{2}{|c|}{ Fecundity } & \multicolumn{2}{|c|}{$\%$ Hatchability } & \multicolumn{2}{|c|}{$\%$ Larval survival } \\
\hline & Mean & S.E. & Mean & S.E. & Mean & S.E. \\
\hline \multicolumn{7}{|c|}{ T. freemani female } \\
\hline FF-F & 66.5 & 9.60 & 81.07 & 3.59 & 89.40 & 3.48 \\
\hline CF-F & 83.3 & 12.77 & 85.61 & 4.60 & 87.62 & 3.33 \\
\hline CC-F & 73.0 & 11.49 & 80.54 & 4.26 & 88.65 & 4.13 \\
\hline \multicolumn{7}{|c|}{ T. castaneum female } \\
\hline $\mathrm{CC}-\mathrm{C}$ & 167.5 & 20.33 & 42.69 & 10.72 & 77.34 & 9.01 \\
\hline $\mathrm{CF}-\mathrm{C}$ & 170.5 & 19.65 & 41.23 & 10.82 & 71.95 & 6.84 \\
\hline FF-C & 64.0 & 5.46 & 12.69 & 2.40 & 43.33 & 7.46 \\
\hline
\end{tabular}

In Figure 1, we show time-course patterns of the numbers of eggs laid per female in the different treatments. The fecundity of $T$. freemani females is highest early and then declines independent of male treatment (Figure 1a). In contrast, T. castaneum females exhibit increasing egg production through day 12 , followed by a slight tapering off after day 16 (Figure 1b). Although the overall pattern is similar within the three different treatments with the $T$. castaneum female, with heterospecific males (FF-C) fecundity is much lower than in either the CF-C or CC-C treatments. Thus, the presence of a conspecific male appears to increase the fecundity of $T$. castaneum females.

No large differences in copulatory rate of conspecific and heterospecific males were observed in our direct observations of mating behaviour. T. freemani females copulated nine times with the $T$. freemani male and eight with the T. castaneum male. To account for the 15 -fold difference in the proportion of hybrids observed in the CF-F treatment, approximately 1 of the 17 copulations should have been with the heterospecific (C) male (binomial probability $<0.00005$ ). While we have not ruled out low levels of premating reproductive isolation, we can rule out premating isolation as an explanation for our results.

$T$. castaneum females were observed to copulate six times with the conspecific male and three with the heterospecific male. This ratio is not significantly different from random mating but is sufficient to account for the discrepancy in the proportion of hybrids in the CF-C treatment. Additional experiments, sequentially offering $T$. castaneum and $T$. freemani males to $T$. castaneum females at 2 day intervals, where male mating speeds and female choice of mate are not at issue, support the interpretation of a postcopulatory mechanism favouring conspecific sperm. When a $T$. castaneum female $(\mathrm{c}-\mathrm{pl})$ is paired with a $T$.

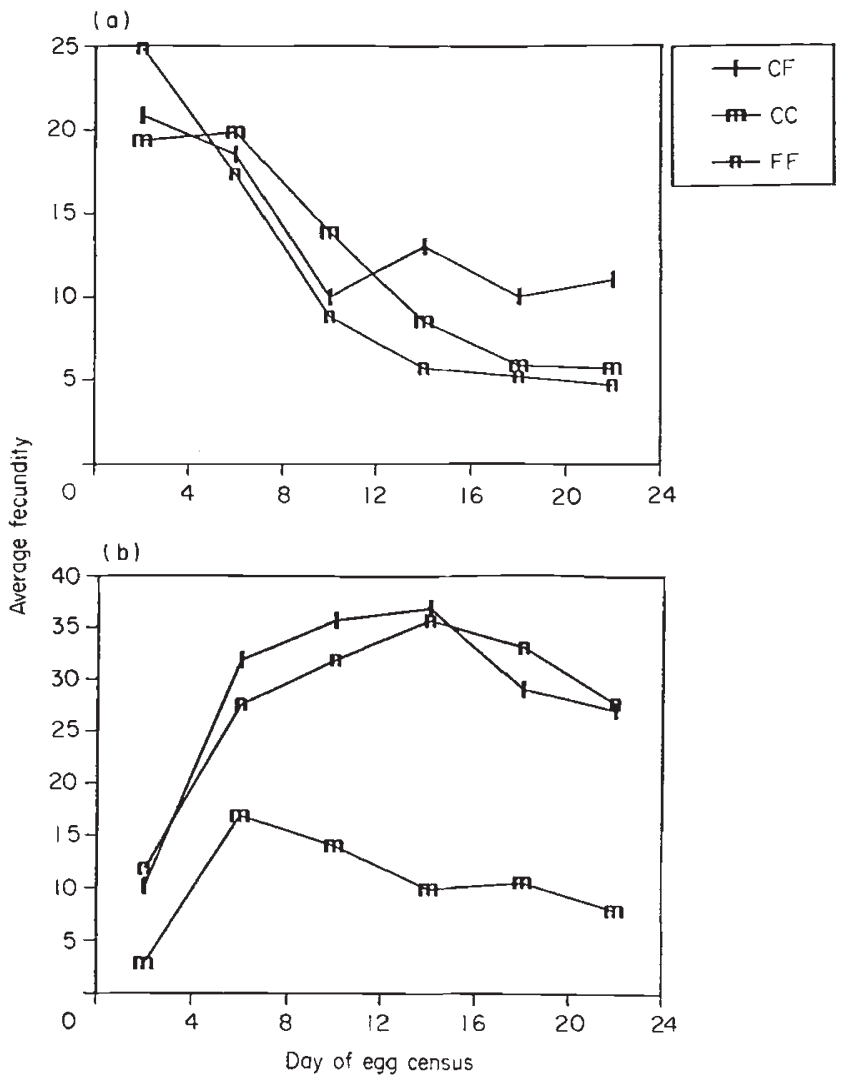

Fig. 1 The number of eggs laid by (a) $T$. freemani and (b) $T$. castaneum females over time under different crossing regimens.

freemani male which is then replaced by a $T$. castaneum male, all of the offspring produced after the replacement are sired by the conspecific male. In contrast, when a $T$. castaneum female is paired with a $T$. castaneum male which is replaced by a $T$. freemani male, no change in paternity is observed: all of the 
offspring are sired by the conspecific male ( $\mathrm{T}$. Robinson and N. Johnson, unpublished data).

\section{Discussion}

The lack of interspecific hybrid offspring in the presence of conspecific males in the CF-F and CF-C treatments, could be due to premating differences in courtship rate, duration or other aspects of precopulatory mate choice by females that favour conspecific males. Our subsequent experiments on mating behaviour and our sequential treatment experiments rule out these premating differences.

We have observed postcopulatory but prezygotic reproductive isolation in flour beetles. We postulate that the lack of hybrids produced in the CF-F and $\mathrm{CF}-\mathrm{C}$ treatments is due to gametic selection favouring the sperm of conspecific males postcopulation. By this we mean that there is a postmating but prezygotic advantage to conspecific sperm within the spermatheca of the female. A variety of mechanisms ranging from sperm recognition (female choice) to interspecific sperm competition (male-male gametic competition) could be responsible (see Thornhill \& Alcock, 1983 for intraspecific analogues).

Our observations suggest a different mechanism from that of the 'insemination reaction' reported for some species of Drosophila (Patterson, 1946). Here, after copulation, the female's vaginal wall greatly increases in size, forming a reaction mass that inhibits fertilization (Patterson, 1946). In heterospecific matings, this reaction effectively blocks fertilization and acts as a postcopulatory but prezygotic isolating mechanism. This occurs whether or not a conspecific male is present. In the homospecific matings, fertilization eventually occurs despite the reaction. See Grant (1983) for tests of other evolutionary explanations of the 'insemination reaction'.

In contrast, fertilization in heterospecific pairings in Tribolium in the absence of conspecific males is normal because, in all time periods, females in the $\mathrm{CC}-\mathrm{F}$ treatment produce progeny numbers comparable to those in the FF-F treatment. Our findings, however, do bear a strong resemblance to interspecific pollen competition in plants (Walsh \& Charlesworth, 1992), where the female stigmatic environment can influence fertilization rates by affecting pollen tube growth rates.

Our results are very similar to those recently reported by Howard \& Gregory (1993). In their studies of two closely related species of crickets in the genus Allonembius, females of each species were given sequential treatments of both species of males. In all of the sequential treatments, the conspecific male sired the vast majority of the offspring although females presented solely with heterospecific males will produce many progeny. Evidence from two genera of grasshoppers (Hewitt et al., 1989; Bella et al., 1992) also suggests that fertilization success and postmating, prezygotic reproductive isolation involves an interaction between male sperm and the female reproductive tract.

There are other possible examples of postmating, prezygotic reproductive isolation. For instance, Grimaldi et al. (1992) have shown that in some interspecific crosses in the Drosophila testacea species subgroup, mating occurs but no sperm is transferred. In no choice tests, Coyne (1993) has shown that $D$. simulans females limit the duration of copulation with $D$. mauritiana males and affect sperm transfer. Our observations and those of Howard \& Gregory (1993) are different in that the isolation is facultative: when given a choice of conspecific and heterospecific males, females produce progeny sired only by the conspecific although they are competent and produce progeny when paired only with heterospecifics. The interspecific progeny numbers and patterns of fecundity that we observed suggest that some type of female choice or male-male competition is occurring within the spermatheca of multiplymated females. If heterospecific matings occur frequently in natural populations of these species, it is possible that this kind of reproductive isolation has been directly selected to prevent the production of sterile hybrids.

\section{Acknowledgements}

We thank Theresa Robinson for assistance in the experiments. We thank the following for comments on and/or discussions of the manuscript: Brian Charlesworth, Deborah Charlesworth, Jerry Coyne, John Jaenike, John Kelly, Alan Molumby, Mohamed Noor, Mike Palopoli, Chung-I Wu and an anonymous referee. This work has been supported by a National Institutes of Health grant (GM 22523) to M.J.W.

\section{References}

BELLA, J. L., BUTLIN, R. K., FERRIS, C. AND HEWITT, G. M. 1992. Assymetrical homogamy and unequal sex ratio from reciprocal mating-order crosses between Chorthippus parallelus subspecies. Heredity, 68, 345-352.

BROWNLEE, A. AND SOKOlOFF, A. 1988. Transmission of Tribolium castaneum (Herbst) mutants to $T$. castaneum- $T$. freemani (Hinton) hybrids (Coleoptera: Tenebrionidae) $J$. Stored Prod. Res., 24, 145-150.

BUTLIN, R. 1987. Speciation by reinforcement. Trends Ecol. Evol., 2, 8-13. 
BUTLIN, R. 1989. Reinforcement of premating isolation. In: Otte, D. and Endler, J. (eds) Speciation and Its Consequences, pp. 158-179. Sinauer Press, Sunderland, MA.

CHARLESWORTH, B., COYNE, J. A. AND BARTON, N. H. 1987. The relative rates of evolution of sex chromosomes and autosomes. Am. Nat., 130, 113-146.

COYNE, J. A. 1992. Genetics and speciation. Nature, 355 , 511-515.

COYNE, J. A. 1993. The genetics of an isolating mechanism between two sibling species of Drosophila. Evolution, 47, 778-789.

COYNE, J. A. AND ORR, H. A. 1989. Patterns of speciation in Drosophila. Evolution, 42, 362-381.

DORZHANSKy, TH. 1937. Genetics and the Origin of Species. Columbia University Press, New York.

DOBZHANSKY, TH. 1940. Speciation as a stage in evolutionary divergence. Am. Nat., 74, 312-321.

DOBZHANSKY, тH. 1970. Genetics and the Evolutionary Process. Columbia University Press, New York.

GRANT, B. 1983. On the relationship between average copulation duration and insemination reaction in the genus Drosophila. Evolution, 37, 854-856.

GRIMALD1, D., JAMES, A. C. AND JAENIKE, J. 1992. Systematics and modes of reproductive isolation in the holarctic Drosophila testacea species group (Diptera: Drosophillidae). Ann. Entomol. Soc. Am., 85, 671-685.

HEWITT, G. M., MASON, P. AND NICHOLS, R. A. 1989. Sperm precedence and homogamy across a hybrid zone in the alpine grasshopper, Podisma pedestris. Heredity, 62, 343-353.

HINTON, H. E. 1948. A synopsis of the genus Tribolium MacLeay, with some remarks on the evolution of its species-groups (Coleoptera: Tenebronidae). Bull. Ent. Res., 39, 13-55.

HOWARD, D. J. 1993. Reinforcement: the origin, dynamics and fate of an evolutionary hypothesis. In: Harrison, R. G. (ed.) Hybrid Zones and the Evolutionary Process, pp. 118-142. Oxford University Press, Oxford.

HOWARD, D. J. AND GREGORY, P. G. 1993. Post-insemination signalling systems and reinforcement. Phil. Trans. R. Soc. Lond. B., 340, 231-236.

JUAN, C., VAZQUEZ, P., RUBIO, J. M., PETITPIERRE, E. AND HEWITT, G. M. 1993. Presence of highly repetitive DNA sequences in Tribolium flour-beetles. Heredity, 70, 1-8.

LITTLEJOHN, M. J. 1981. Reproductive isolation: a critical review. In: Atchley, W. R. and Woodruff, D. (eds) Evolution and Speciation Cambridge University Press, Cambridge.

MAYr, E. 1963. Animal Species and Evolution. Harvard University Press, Cambridge, MA.

MULLER, H. J. 1942. Isolating mechanisms, evolution and temperature. Biol. Symp., 6, 71-125.

PARK, T. 1933. Studies in population physiology. II. Factors regulating initial growth of Tribolium confusum populations. J. Exp. Zool., 65, 17-42.

PARK, T. 1937. The inheritance of the mutation 'pearl' in the flour beetle Tribolium castaneum Herbst. Am. Nat., 71, $143-157$

PARK, T., LESLIE, P. H. AND MERTZ, D. B. 1964. Genetic strains and competition in populations of Tribolium. Phys. Zool., 37, 97-161.

PATERSON, H. E. H. 1978. More evidence against speciation by reinforcement. S. Afr. J. Sci., 74, 369-371.

PatTerson, J. T. 1946. A new isolating mechanism in Drosophila. Proc. Nat. Acad. Sci. U.S.A., 32, 202-208.

SHRODE, R. R. 1960. Evidence that mating is random in $T$. castaneum. Tribolium Inf. Bull., 3, 15-16.

soKolofF, A. 1974. The Biology of Tribolium, Vol. 2. Clarendon Press, Oxford.

THORNHILL, R. AND ALCOCK, J. 1983. The Evolution of Insect Mating Systems. Harvard University Press, Cambridge, MA.

WADE, M. J. 1976. Group selection among laboratory populations of Tribolium. Proc. Nat. Acad. Sci. U.S.A., 73, 4604-4607.

WADE, M. J. 1977. An experimental study of group selection. Evolution, 31, 134-153.

WADE, M. J. AND GOODNIGHT, C. J. 1991 . Wright's shifting balance theory: an experimental study. Science, 253, 1015-1018.

WADE, M. J. AND JOHNSON, N. A. 1993. Reproductive isolation between two species of flour beetles, Tribolium castaneum and $T$. freemani: variation within and among geographical populations of $T$. castaneum. Heredity, 72, 155-162.

WALSH, N. E. AND CHARLESWORTH, D. 1992. Evolutionary interpretations of differences in pollen tube growth rates. Quart. Rev. Biol., 67, 19-36.

WU, C.-I. AND DAVIS, A. W. 1993. Evolution of postmating reproductive isolation: the composite nature of Haldane's rule and its genetic bases. Am. Nat., 142, 189-212. 